\title{
Fractional quantum Hall states of a Bose gas with spin-orbit coupling
}

\author{
T. Graß ${ }^{1}$, B. Juliá-Díaz ${ }^{1}$, M. Burrello ${ }^{2}$, and M. Lewenstein ${ }^{1,3}$ \\ ${ }^{1}$ ICFO-Institut de Ciències Fotòniques, Parc Mediterrani de la Tecnologia, 08860 \\ Barcelona, Spain \\ ${ }^{2}$ Instituut-Lorentz, Universiteit Leiden, P.O. Box 9506, 2300 RA Leiden, The \\ Netherlands \\ ${ }^{3}$ ICREA-Institució Catalana de Recerca i Estudis Avançats, 08010 Barcelona, Spain \\ E-mail: tobias.grass@icfo.es
}

\begin{abstract}
We study the fractional quantum Hall phases of a pseudospin-1/2 Bose gas in an artificial gauge field. In addition to an external magnetic field, the gauge field also mimics an intrinsic spin-orbit coupling of the Rashba type. While the spin degeneracy of the Landau levels is lifted by the spin-orbit coupling, the crossing of two Landau levels at certain coupling strengths gives rise to a new degeneracy. We therefore take into account two Landau levels, and perform exact diagonalization of the many-body Hamiltonian. We study and characterize the quantum Hall phases which occur in the vicinity of the degeneracy point. Notably, we describe the different states appearing at the Laughlin fillings, $\nu=1 / 2$ and $\nu=1 / 4$. While for these filling factors incompressible phases disappear at the degeneracy point, denser systems at $\nu=3 / 2$ and $\nu=2$ are found to be clearly gapped. For filling factors $\nu=2 / 3$ and $\nu=4 / 3$, we discuss the connection of the exact ground state to the non-Abelian spin singlet states, obtained as the ground state of $k+1$ body contact interactions.

PACS numbers: $67.85 . \mathrm{Fg}, 73.43 .-\mathrm{f}$
\end{abstract}

Keywords: Quantum Hall states. Artificial gauge fields. Spin-orbit coupling. Ultracold bosons. 


\section{Introduction}

Cold atoms provide versatile and highly controllable quantum systems. This makes them particularly useful as quantum simulators, with plenty of applications which stem from their complicated many-body behavior. Cold atoms have been used to explore important open problems in solid state physics. A noteworthy example is the physics emerging from strongly correlated electronic systems, which has fascinated researchers for decades. Due to its topological nature and the exotic anyonic excitations, fractional quantum Hall (FQH) systems are particularly interesting candidates for a quantum simulation with cold atoms [1, 2, 3, 4].

A major difference between electrons and atoms, however, is the atoms' lack of electric charge. This makes them insensitive to external magnetic fields, which would hinder exploring the vast field of quantum Hall physics. Different proposals to overcome this have been made: In principle, rotating the atomic system is sufficient to mimic the Lorentz force by mean of the Coriolis force [5, 6] and bring the system into quantum Hall phases [7, 8, 9]. However, experimental attempts to reach this regime by rotating the cloud have not been entirely successful [10, 11, 12, 13, 14. Alternatively, the magnetic field can be mimicked by dressing the atoms with laser fields in such a way that the lowest dressed state obtains a space-dependent phase factor. Properly chosen, this phase factor acts as if the atoms were moving like charged particles in a magnetic field [15, 16, 17]. A Raman dressing of ${ }^{87} \mathrm{Rb}$ atoms has been used to generate an artificial electric field [18]. Later on, this scheme has successfully mimicked an artificial magnetic field and visibly induced vortices into the atomic cloud [19]. Artificial gauge fields have also been realized in optical lattices, by means of laser dressing [20, 21] or shaking of the lattice [22].

These schemes allow for simulating the behavior of charged spinless (or spinpolarized) particles. The spin degree of freedom of electrons might play a role in fractional quantum Hall setups, as the strong magnetic field does not necessarily fully polarize the electron spin due to the small gyromagnetic ratio in many solid materials. Allowing the atoms to be in two internal states, one might try to mimic spin- $1 / 2$ particles like electrons, but again the lack of charge will cause significant differences between this pseudospin, and the real spin of the electrons. The latter interacts with a magnetic field which is induced by the motion of the charged electron through the electric field of the nuclei. This effect, called Rashba spin-orbit coupling [23], is absent in cold atoms. Ways to overcome this have been proposed recently. The laser dressing of atoms can be designed in such a way that it not only implements the effect of a magnetic field, but also of a spin-orbit coupling [24, 25, 26]. Mathematically, spin-orbit coupling and magnetic fields can be treated on the same footing: While the magnetic field can be taken into account through a minimally coupled vector potential $\boldsymbol{A}$, i.e. a term $\boldsymbol{p} \cdot \boldsymbol{A}$ in the Hamiltonian, the spin-orbit coupling has the form $\boldsymbol{p} \cdot \boldsymbol{\sigma}$, where $\boldsymbol{\sigma}=\left(\sigma_{x}, \sigma_{y}, \sigma_{z}\right)^{T}$, a vector of Pauli matrices. This makes the spin-orbit coupling equivalent to gauge fields, which in the case of spin-1/2 belong to the $\mathrm{SU}(2)$ group, and are thus in general referred to as non-Abelian gauge fields. In pioneering experiments, spin-orbit coupling 
has been synthesized experimentally for ${ }^{87} \mathrm{Rb}$ [27, 28], using a Raman dressing similar to the one of Refs. [18, 19]. These experiments have attracted a great deal of attention, and the impact of spin-orbit coupling on the condensation properties of a Bose gas has been studied theoretically [29, 30, 31, 32, 33, 34, 35]. Proposals to synthesize spin-orbit coupling for atoms in optical lattices [36, 37, also motivate the study of Bose-Hubbard models with spin-orbit coupling [38, 39, 40].

A different scenario is encountered in the presence of both a spin-orbit coupling and a strong artificial magnetic field. The latter induces strong correlations, producing fractional quantum Hall phases which in turn may be qualitatively affected by the spinorbit coupling. Its influence could perfectly be revealed by a cold atom experiment which combines the artificial spin-orbit coupling and the artificial magnetic field, and allows to freely tune the coupling strength. The closest connection to the electronic fractional quantum Hall effect can be made by considering fermionic atoms with dipolar interactions [41].

In this paper, we consider the experimentally more feasible scenario of bosonic atoms with short-range interactions. They are confined to a plane, or, as we will assume periodic boundary conditions, to a torus. The bosons are subjected to an artificial magnetic field perpendicular to the plane, and we assume a Rashba coupling between an effective pseudospin- $1 / 2$ degree of freedom and the external motion of the atoms. It has recently been shown that in the absence of spin-orbit coupling but within an artificial magnetic field a two-component Bose gas forms spin singlets [42, 43]. The strongly correlated regime is then described by a series of states with non-Abelian anyon excitations (non-Abelian spin singlet or NASS states) [44, 45]. It has also been shown that via a Rashba-type spin-orbit coupling a certain spin polarization is favored, resulting in spin polarized quantum Hall phases [46, 47] which are derived from the Read-Rezayi series [48]. Distinct quantum Hall phases, however, have been predicted for particular spin-orbit coupling strengths which correspond to a degeneracy between two Landau levels [46, 47]. Numerical studies restricted to a single Landau level have been able to back the occurrence of the Read-Rezayi-like phases [49, 50]. For the interesting configuration at the degeneracy points, gapped phases at the NASS filling factors have been numerically proven recently [42.

We undergo a complete numerical study, complementing the results presented in [42, thus taking into account two Landau levels. Performing exact diagonalization with such an increased basis, our study remains valid also at the degeneracy points. We start with a detailed description of the system in Sec. 2. Next, we solve the single-particle problem in Sec. 3. Afterward, we turn to the many-body problem in Sec. 4, calculating the interaction matrix elements. With this we can perform the exact diagonalization, yielding the results presented in Secs. 5 and 6. Our main findings are summarized in Sec. 7 . 


\section{The system}

We consider a pseudospin-1/2 Bose gas in two dimensions, described by a single-particle Hamiltonian

$$
H_{i}=\frac{1}{2 M}\left[\boldsymbol{p}_{i} \mathbf{1}_{2}+\boldsymbol{A}\left(\boldsymbol{r}_{i}\right)\right]^{2},
$$

with $\mathbf{1}_{2}$ being the $2 \times 2$ identity matrix. An artificial gauge potential $\boldsymbol{A}$ mimics a constant, perpendicular magnetic field and a coupling between the pseudospin and the external motion. For convenience, we choose this coupling to be of the Rashba-type. In the Landau gauge, the vector potential reads

$$
\boldsymbol{A}=B(0, x, 0) \mathbf{1}_{2}+q\left(\sigma_{x}, \sigma_{y}, 0\right) .
$$

Here, $B$ is the magnetic field strength, $q$ controls the strength of the spin-orbit coupling, and $\sigma_{i}$ are Pauli matrices. Both contributions to this gauge potential have already been engineered experimentally separately: An artificial magnetic field of this form (i.e. $q=0$ ) has been realized through an atom-laser dressing in Bose-Einstein condensates without pseudospin degree of freedom [19], and through rotation in two-component systems [11, 12]. A similar spin-orbit coupling has been achieved experimentally by a Raman coupling of three atomic states with two lasers [27, 28]. A theory proposal of how to achieve a gauge potential combining both the magnetic and the spin-orbit part has been given in Ref. [46, 47]. Here, the spin-orbit term arises due to a tripod coupling of three atomic ground states to one excited state by three laser fields. A review of different proposals is given in Ref. [15].

All schemes have in common that they effectively consider dressed atoms, that is superpositions of different bare atomic states. It is in the basis of dressed states that the single-particle Hamiltonian takes the desired form of Eq. (1). While equivalent on the single-particle level, the different proposals might require different descriptions for the particles' interactions. Tripod schemes with different laser configurations, though mimicking the same spin-orbit coupling, have been shown to support different mean-field phases due to differences in the interaction term [51]. It has also experimentally been shown that the interactions between dressed atoms might become long-range, though the bare atoms interact via $s$-wave scattering [28]. Also if the interaction potential of the dressed atoms effectively remains a contact potential, the scattering lengths may be modified. In the first experimental realization of spin-orbit coupling, the strength of interactions between different pseudospin states behaved linearly with the effective spinorbit coupling strength, which caused a quantum phase transition between a pseudospin-

mixed phase and a pseudospin-separated phase upon tuning the spin-orbit coupling strength [27].

Thus, the choice of interactions is a delicate issue. In this paper, we consider two-body contact interactions between the dressed atoms.

$$
V\left(\boldsymbol{r}_{i}, \boldsymbol{r}_{j}\right)=\frac{\hbar^{2}}{M} \delta^{(2)}\left(\boldsymbol{r}_{i}-\boldsymbol{r}_{j}\right)\left(\begin{array}{cc}
g_{\uparrow \uparrow} & g_{\uparrow \downarrow} \\
g_{\uparrow \downarrow} & g_{\downarrow \downarrow}
\end{array}\right) .
$$


Such a description is appropriate for the pioneering experiment in Ref. [27]. In this experiment, ${ }^{87} \mathrm{Rb}$ has been used with nearly equal scattering lengths in and between different hyperfine states. Also, for weak or moderate spin-orbit coupling strength the scheme of Ref. [27] does not significantly alter the effective scattering lengths for the dressed atoms. It is thus convenient to assume $\mathrm{SU}(2)$ symmetry, that is, $g_{\uparrow \uparrow}=g_{\downarrow \downarrow}=g_{\uparrow \downarrow} \equiv g$.

Previous studies have performed numerical diagonalization of pseudospin-1/2 systems without spin-orbit coupling $(q=0)$ [42, 43]. We note that in this case, the pseudospin polarization $S$ defined as the difference $N_{\uparrow}-N_{\downarrow}$ of up- and down-spin particles is a conserved quantity. This reduces the size of the Hilbert space, and yields eigenstates of well-defined polarization. For SU(2)-symmetric interactions, at almost all filling factors the ground state is found to be a pseudospin singlet, $S=0$. For bosons interacting via a two-body contact potential, eigenstates with zero interaction energy exist up to a filling factor $\nu=2 / 3$. Here the (221) Halperin state becomes the unique zero interaction energy state, if one does not take into account edge excitations, as is the case within our numerics performed on a torus. The wave function of the (221) Halperin state on a disk reads [52]

$$
\Psi_{\mathrm{H}}=\prod_{i<j}^{N_{\uparrow}}\left(z_{i \uparrow}-z_{j \uparrow}\right)^{2} \prod_{k<l}^{N_{\downarrow}}\left(z_{k \downarrow}-z_{l \downarrow}\right)^{2} \prod_{i, k}\left(z_{i \uparrow}-z_{k \downarrow}\right) \mathrm{e}^{-\sum \frac{\left|z_{i}\right|^{2}}{4}},
$$

where we have introduced the complex variables, $z_{j}=x_{j}-i y_{j}$.

This Halperin state is part of a series of states occurring at filling factors $\nu=$ $2 k / 3$, defined as the maximum-filled zero-energy eigenstates of a $(k+1)$-body contact interaction. This series is commonly known as the NASS series, since the states with $k \geq 2$ support non-Abelian anyonic excitations [44, 45]. As pointed out in Refs. [42, 43], the non-Abelian phase at filling $\nu=4 / 3$ is realized even in systems with a two-body contact potential.

Also systems with finite $q$ have been considered numerically [49, 50]. The lowest Landau level approximation which has been made in these studies is valid within broad ranges of $q$ with sufficiently large Landau level gaps. These studies have shown that in these regimes the spin-polarized fractional quantum Hall states like the Laughlin state [1] or Moore-Read state [3] are relevant, if one maps them into the Landau level structure of the spin-orbit coupled system according to Eq. (18). We will demonstrate this in more detail in Secs. 5 and 6 for the bosonic Laughlin state at filling factor $\nu=1 / 2$, investigating its fate when passing through the degeneracy point $q^{2}=3 B$, and for other relevant filling factors which may present incompressible phases, respectively.

\section{Single particle Hamiltonian: Landau level structure}

Prior to solving the many-body problem by numerical diagonalization, it is useful to solve the single-particle Hamiltonian. For the spin-orbit coupling of Eq. (2), this has been done in Refs. [46, 47], yielding a Landau level structure which is simply a 
combination of two Landau levels from the quantum Hall system without spin-orbit coupling. While they consider a system on a disk, for our numerical study it is advantageous to work in an edge-less geometry, as it allows to study bulk properties even for small system sizes. We thus choose a rectangle of sizes $a$ and $b$ with periodic boundary conditions, that is a torus, and perform the analog derivation for the Landau level structure.

For convenience, we choose units such that $\hbar=1$ and $M=1 / 2$, and define the following ladder operators:

$$
\begin{aligned}
c & \equiv \frac{1}{\sqrt{2 B}}\left(p_{x}-i p_{y}-i B x\right), \\
c^{\dagger} & \equiv \frac{1}{\sqrt{2 B}}\left(p_{x}+i p_{y}+i B x\right),
\end{aligned}
$$

with $\left[c, c^{\dagger}\right]=1$ and $[c, c]=0$. The single-particle Hamiltonian can now be expressed in terms of these operators

$$
H_{i}=\left(\begin{array}{cc}
2 B\left(c_{i}^{\dagger} c_{i}+\frac{1}{2}\right)+2 q^{2} & 2 \sqrt{2 B} q c_{i} \\
2 \sqrt{2 B} q c_{i}^{\dagger} & 2 B\left(c_{i}^{\dagger} c_{i}+\frac{1}{2}\right)+2 q^{2}
\end{array}\right) .
$$

The diagonal part has the form of a harmonic oscillator, yielding the standard Landau levels $\tilde{\Psi}_{n, \kappa}(x, y)=\exp (i \kappa y) \Phi_{n, \kappa}(x)$ and

$$
\Phi_{n, \kappa}(x)=e^{-B(x+\kappa / B)^{2} / 2} \quad H_{n}[\sqrt{B}(x+\kappa / B)]
$$

with $H_{n}$ the Hermite polynomials. The energy quantum number $n$ denotes the Landau level. Applying the periodic boundary conditions, as done in Ref. [53], $\kappa$ is restricted to integer multiples of $2 \pi / b$. This yields a second quantum number $j \in \mathbb{Z}$ via the definition $X_{j} \equiv \kappa / B=2 \pi j /(b B)$. The quantity $X_{j}$ can be interpreted as a displacement of the harmonic oscillator in the $x$-direction. To ensure the periodicity of the wave function in $x$, with period $a$, we can sum over all displacements $X_{j}+k a$ with $k$ an integer, $\Psi_{n, j}(x, y) \equiv \sum_{k=-\infty}^{\infty} \tilde{\Psi}_{n, B\left(X_{j}+k a\right)}(x, y)$. This will not affect the $b$-periodicity in $y$, if $a b=2 \pi \lambda^{2} N_{\Phi}$ with $N_{\Phi}$ an integer. To prevent double-counting, we must restrict $j$ to $1 \leq j \leq N_{\Phi}$. We find that $N_{\Phi}$ quantizes the magnetic field, and can thus be interpreted the number of magnetic fluxes within the unit cell. It is connected to the particle number $N$ through the filling factor

$$
\nu=\frac{N}{N_{\Phi}} \equiv \frac{P}{Q}
$$

where we also have introduced the co-prime integers $P$ and $Q$. Introducing the unitless variables $x^{\prime}=x / \lambda$ and $y^{\prime}=y / \lambda$, with $\lambda=1 / \sqrt{B}$, we finally arrive at the wave functions of Ref. [53]. In the lowest Landau level (i.e. for $n=0$ ), they read

$$
\Psi_{0, j}=\left(2 \pi^{2} N_{\Phi}^{3} \frac{a}{b}\right)^{-1 / 4} \mathrm{e}^{-\frac{y^{\prime 2}}{2}-i x^{\prime} y^{\prime}} \theta_{3}\left[\frac{j \pi}{N_{\Phi}}+\sqrt{\frac{\pi}{2 N_{\Phi} a / b}} z, e^{-\frac{\pi}{N_{\Phi} a / b}}\right] .
$$

Here, $\theta_{3}$ is an elliptic $\theta$-function. The excited states can easily be obtained by applying the ladder operator $c^{\dagger}, \sqrt{n+1} \Psi_{n+1, j}=c^{\dagger} \Psi_{n, j}$. 

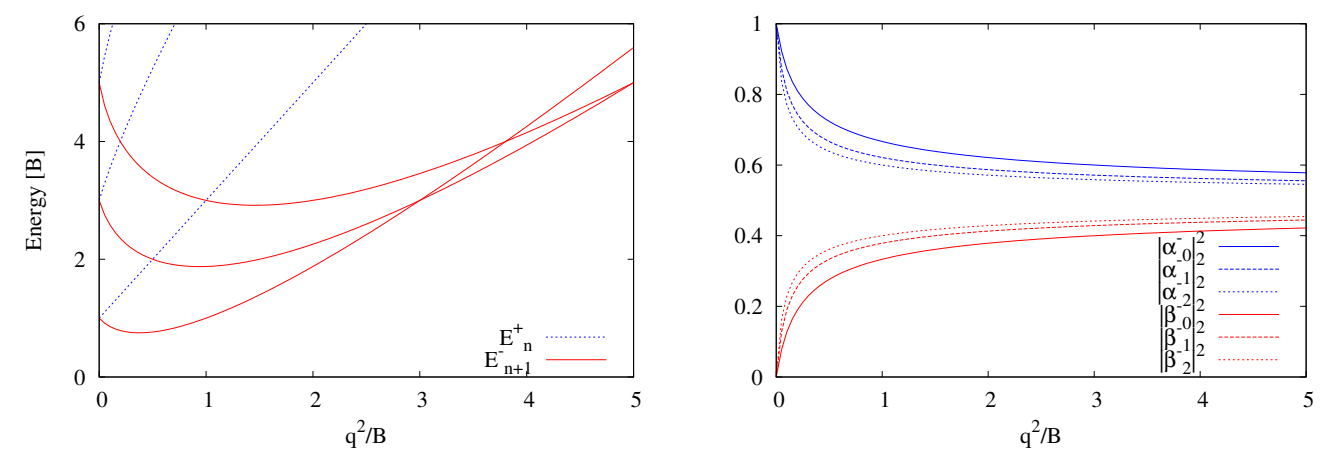

Figure 1. Left: First eigenvalues of the single particle Hamiltonian, $E_{n}^{ \pm}$as a function of $q^{2} / B$, see Eq. (13). Right: Weights of the two components of each eigenvector of the single particle Hamiltonian for the "-" states, $\alpha_{n}^{-}$, and $\beta_{n}^{-}$as a function of $q^{2} / B$, see Eq. (16).

Without spin-orbit coupling, i.e. for $q=0$ in Eq. (7), the wave function

$$
\Psi_{0, j}^{q=0} \equiv \Psi_{0, j}(\alpha|\uparrow\rangle+\beta|\downarrow\rangle) \text {. }
$$

with $|\alpha|^{2}+|\beta|^{2}=1$ describes the lowest Landau level. Thus, apart from the degeneracy in $j$, we have an $\mathrm{SU}(2)$ degeneracy due to the pseudospin-1/2 degree of freedom.

For finite $q$, we have to take into account higher Landau levels, as the off-diagonal elements in Eq. (7) provide a Jaynes-Cummings-type coupling. Thus, the eigenspinors must be of the form

$$
\Psi_{n, j}^{ \pm} \equiv\left(\alpha_{n}^{ \pm} \Psi_{n, j}, \beta_{n}^{ \pm} \Psi_{n+1, j}\right)^{T},
$$

where $\alpha_{n}^{ \pm}$and $\beta_{n}^{ \pm}$are functions of $q$. The single-particle spectrum is found to be the same as in Refs. [46, 47], as expected due to gauge invariance. It reads

$$
E_{n}^{ \pm}=2 B(n+1)+2 q^{2} \pm \sqrt{B^{2}+8 B q^{2}(n+1)} .
$$

In Fig. 11 we present the lowest eigenvalues $E_{n}^{ \pm}$.

Since the eigensolutions corresponding to $E_{n}^{+}$are higher than the lowest $E_{n}^{-}$ solutions, we can restrict ourselves to the latter. Their coefficients $\alpha_{n}^{-}$and $\beta_{n}^{-}$read

$$
\begin{aligned}
\alpha_{n}^{-} & =\mathcal{N}\left(B+2 q \sqrt{2 B(n+1)}+\sqrt{B^{2}+8 B q^{2}(n+1)}\right), \\
\beta_{n}^{-} & =\mathcal{N}\left(B-2 q \sqrt{2 B(n+1)}-\sqrt{B^{2}+8 B q^{2}(n+1)}\right), \\
\mathcal{N} & =\frac{1}{2}\left(B^{2}+8 B q^{2}(n+1)+2 q \sqrt{2 B(n+1)}\right. \\
& \left.\times \sqrt{B^{2}+8 B q^{2}(n+1)}\right)^{-1 / 2} .
\end{aligned}
$$

In Fig. 1 we plot the value of the coefficients $\alpha_{n}^{-}$and $\beta_{n}^{-}$as a function of the spin-orbit coupling strength, $q$. The lower spin component quickly acquires weight, measured by $|\beta|^{2}$, as the spin-orbit coupling is turned on.

We shall distinguish two cases, as seen in Fig. 1, or Eq. (13): 1) For broad ranges of $q$, the ground state is given by a single $E_{n}^{-}$level. Thus, the pseudospin degeneracy 
of the system with $q=0$ is lifted, and the system is effectively spin-polarized. 2) The second case is restricted to separate points at $q^{2} / B=2 n+3$, where two solutions, $E_{n}^{-}$ and $E_{n+1}^{-}$, become degenerate. This scenario, with a two-fold degenerate single-particle ground state, is formally similar to the spin-degenerate case of Eq. (11). We can define a pseudospin-1/2 spinor as,

$$
\left(\begin{array}{c}
\gamma \\
\delta
\end{array}\right) \equiv \gamma \boldsymbol{\Psi}_{0, j}^{-}+\delta \boldsymbol{\Psi}_{1, j}^{-} \quad \text { with }|\gamma|^{2}+|\delta|^{2}=1 .
$$

The connection between these two Landau levels of the spin-orbit coupled system and the $q=0$, spin-degenerate lowest Landau levels, is made through the bijective mapping

$$
\begin{gathered}
|0, j, \uparrow\rangle \mapsto \Psi_{0, j}^{-} \equiv \alpha_{0}^{-}|0, j, \uparrow\rangle+\beta_{0}^{-}|1, j, \downarrow\rangle \\
|0, j, \downarrow\rangle \mapsto \Psi_{1, j}^{-} \equiv \alpha_{1}^{-}|1, j, \uparrow\rangle+\beta_{1}^{-}|2, j, \downarrow\rangle .
\end{gathered}
$$

For brevity, in the following we denote these two levels by $|n=0, j\rangle$ and $|n=1, j\rangle$. In our numerical calculations, we will explicitly take into account these two levels, as we focus on configurations where $q^{2} / B$ is close to the first critical value of 3 .

\section{Many-body Hamiltonian}

From the single-particle states, we easily construct many-body Fock states

$$
\left|n_{n=0, j=1}, \cdots, n_{n=0, j=N_{\Phi}}, n_{n=1, j=1}, \cdots, n_{n=1, j=N_{\Phi}}\right\rangle,
$$

and we can express the Hamiltonian in terms of annihilation and creation operators, $\hat{\mathrm{b}}_{n j}^{\dagger}$ and $\hat{\mathrm{b}}_{n j}$. These obey bosonic commutation relations and act on the occupation number $n_{n j}$ in the usual way. The Hamiltonian reads in this notation:

$$
\mathcal{H}=\sum_{n, j} E_{n}^{-} \hat{\mathrm{b}}_{n j}^{\dagger} \hat{\mathrm{b}}_{n j}+\sum_{\{n, j\}} V_{\{n, j\}} \hat{\mathrm{b}}_{n_{1} j_{1}}^{\dagger} \hat{\mathrm{b}}_{n_{2} j_{2}}^{\dagger} \hat{\mathrm{b}}_{n_{3} j_{3}} \hat{\mathrm{b}}_{n_{4} j_{4}} .
$$

The interaction matrix elements $V_{\{n, j\}}$,

$$
V_{\{n, j\}}=\left\langle n_{1}, j_{1}\left|\left\langle n_{2}, j_{2}|V| n_{3}, j_{3}\right\rangle\right| n_{4}, j_{4}\right\rangle
$$

with $V$ given in Eq. (3), and $\{n, j\}$ denoting the set of quantum numbers $n_{1}, \ldots, n_{4}$ and $j_{1}, \ldots, j_{4}$, are given in Appendix A.

Before numerically diagonalizing the Hamiltonian $\mathcal{H}$ of Eq. (21), we shall exploit the translational symmetry of the system on a torus. This symmetry, fully discovered by F. D. M. Haldane [54], yields a many-body basis where states are characterized by a "Haldane momentum" $\boldsymbol{K}=\left(K_{x}, K_{y}\right)$ which is conserved by the Hamiltonian. A comprehensive recipe for constructing this basis can be found in Ref. [55]. The main idea is to divide the many-body states into equivalence classes: An $N$-particle state characterized by the quantum numbers $\left\{j_{1}, \ldots, j_{N}\right\}$ (and the $n$ quantum numbers which here do not matter) can by translation along $x$ be transformed into states with $\left\{j_{1}-q m, \cdots, j_{N}-q m\right\}$, where $q$ is defined in Eq. (9), and $m$ is an integer. It runs from 0 to some maximum value $c_{\eta}$, where $\left\{j_{1}, \ldots, j_{N}\right\}=\left\{j_{1}-c_{\eta} m, \cdots, j_{N}-c_{\eta} m\right\}$ due to the definition of $j$ modulo $N_{\Phi}$. This set of $c_{\eta}$ different states forms an equivalence class 
which we label by $\eta$. We denote each state by $|\eta, m\rangle$. For each class $\eta$, the translational symmetric eigenstates are found as superpositions which can be characterized a quantum numbers $\tilde{J}$ running from 0 to $N_{\Phi} / Q-1$ :

$$
|\eta,(\tilde{J}, J)\rangle \equiv \frac{1}{\sqrt{c_{\eta}}} \sum_{k=0}^{c_{\eta}-1} \exp \left(\frac{2 \pi i \tilde{J} k}{c_{\eta}}\right)|\eta, k\rangle .
$$

A second quantum number $J$ is given by $J \equiv j_{i} \bmod \mathrm{N}_{\Phi}$. Both quantum numbers $\tilde{J}$ and $J$ can be related to the Haldane pseudomomentum $\boldsymbol{K}$ :

$$
\boldsymbol{K} \lambda=\sqrt{\frac{2 \pi b}{N_{\Phi} a}}\left(\tilde{J}-\tilde{J}_{0}, \frac{a}{b}\left(J-J_{0}\right)\right) .
$$

The quantum numbers of zero pseudomomentum are defined as the point in the Brillouin zone with highest symmetry. In most cases, $\left(\tilde{J}_{0}, J_{0}\right)=(0,0)$, but if $N$ is even and $P$ and $Q$ are both odd, other values become possible (cf. Ref. [55]). The numerical diagonalization is then performed within a Hilbert space with fixed filling factor $\nu$ and fixed pseudomomentum $\boldsymbol{K}$.

\section{The fate of the Laughlin state at $\nu=1 / 2$}

The Laughlin state at filling $\nu=1 / Q$ is described on a disk by a wave function:

$$
\Psi_{\mathrm{L}}=\prod_{i<j}\left(z_{i}-z_{j}\right)^{Q} \mathrm{e}^{-\sum_{i}\left|z_{i}\right|^{2} / 4},
$$

For $Q=2$, it constitutes the highest density eigenstate of a spinless or spin-polarized system of bosons interacting via a two-body contact potential, since its wave function becomes zero whenever two particles coincide. We can thus easily find its secondquantized expression by exact diagonalization. Although performing the mapping into the spin-orbit coupled Landau levels according to Eqs. (18) and (19) corresponds to simply redefining the basis of the Hilbert space, this process has non-trivial physical implications. Since the Laughlin state is a spinless state there is an arbitrariness in applying the mapping of either Eq. (18) into the first or Eq. (19) into the second Landau level. The effect of this mapping to higher Landau levels induces a sort of effective long range interaction [50] such that the $n u=1 / 2$ Laughlin state acquires in general a positive interaction energy. This is due to the fact that in the spin-orbit coupled Landau level the corresponding wave function does not vanish when $z_{i} \rightarrow z_{j}$.

Let us first investigate in which regimes of the spin-orbit coupling strength such projected Laughlin states describe well the ground state at filling factor $\nu=1 / 2$. Afterwards, we will consider Laughlin states at smaller filling factors, which remain states of zero interaction energy in the spin-orbit coupled system.

\subsection{Below the first degeneracy point: $0<q^{2} / B<3$}

For $q^{2}<3 B$, the levels $|n=0, j\rangle$ are the lowest single-particle states, see Fig. 1, thus we expect the ground state to be given by the projection into this manifold, 


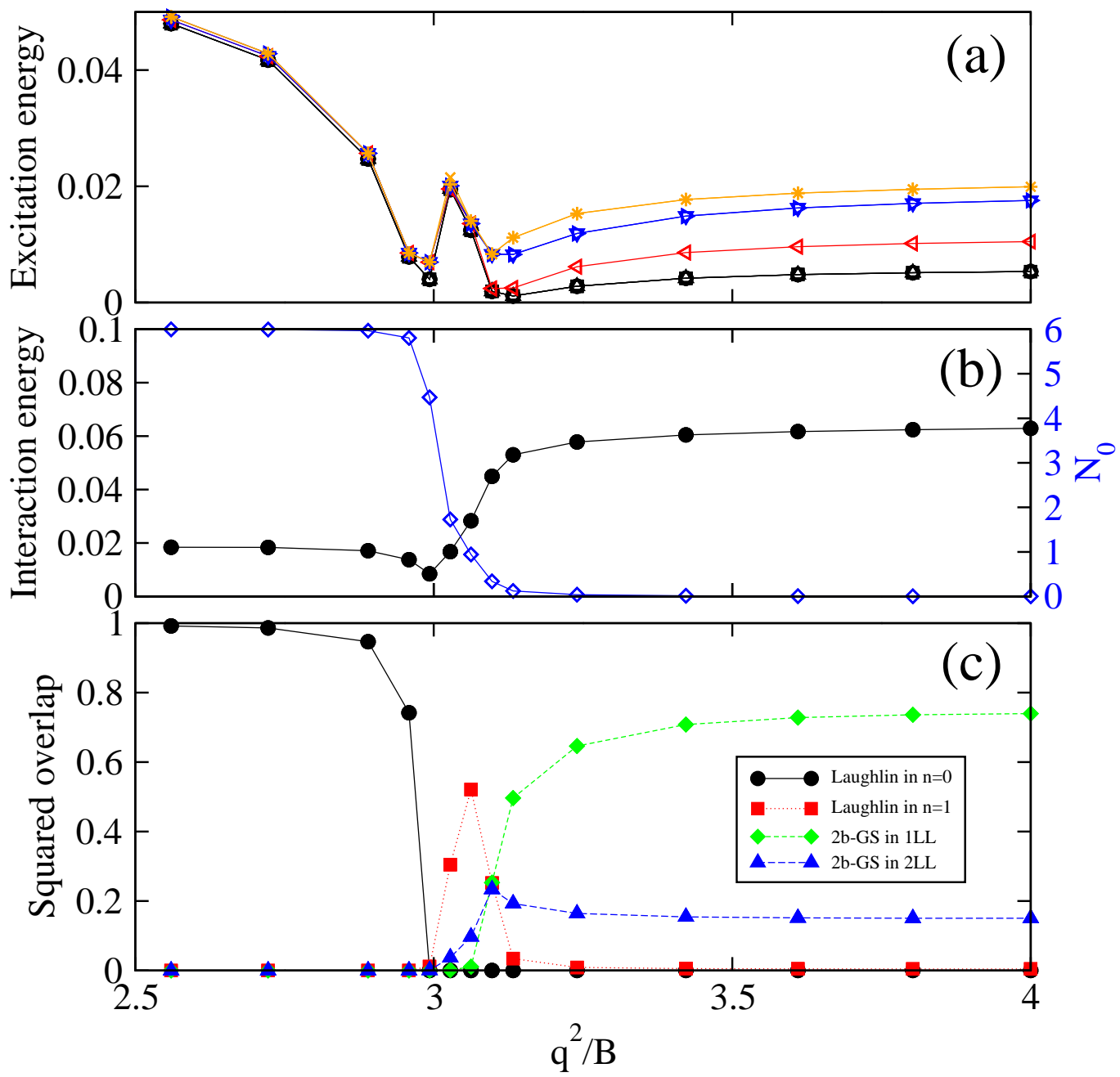

Figure 2. Properties of the system as a function of the strength of the spin-orbit interaction, $q^{2} / B$. (a) Low-lying excitation energies. (b) (left scale) Interaction energy of the ground state. (right scale) Occupation of $n=0$ level in the ground state. (c) Overlap of ground state with different test states as further explained in the text: Laughlin projections, and ground states of two-body contact interaction in the first and second excited LL. The system size is $N=6$ at half filling, with spin-independent interactions $g_{s_{1} s_{2}}=0.2$. Energies are in units of $g B$.

following Eq. (18). As shown in Fig. 2 (c), the overlap of the true ground state with this projected Laughlin state is approximately 1, if we are sufficiently far below the degeneracy point. However, it quickly decreases to 0 when approaching the degeneracy point. Simultaneously, the $n=0$ Landau level is depleted, see Fig. 2 (b).

In Fig. 2 (a), we plot the low-lying excitation energies as a function of $q^{2} / B$. This shows that the projected Laughlin phase is gapped. The gap, however, vanishes when approaching the degeneracy point. In Fig. 2 (b), the interaction energy of the ground state is plotted. The projected Laughlin state has a finite interaction energy, due to interactions within the lower component and between the components. A sudden 
decrease in the interaction energy is observed when the degeneracy point is approached.

The numerical results shown in Fig. 2 reflect the situation of $\mathrm{SU}(2)$ symmetric interactions. The value for the interaction strengths defines the interval in $q$ where the system significantly occupies the $n=1$ level. For the relatively small value $g=0.2$ in Fig. 2 (c), the drop in the overlap curve appears to be quite sharp. We can extend it over a larger region by choosing a stronger interaction. We have also investigated an interaction term, where $g_{\uparrow \uparrow}=g_{\downarrow \downarrow}$ and $g_{\uparrow \downarrow}=0$. Qualitatively, this yields the same results as in the $\mathrm{SU}(2)$-symmetric case for $0<q^{2}<3 B$.

The problem becomes analytically solvable if we choose $g_{\uparrow \uparrow}=g_{\downarrow \downarrow}=0$ and $g_{\uparrow \downarrow}=g>0$ [47]. In this case, the Laughlin state projected into the $n=0$ Landau level is a zero-energy eigenstate of the two-body contact potential, and thus the true ground state below the first degeneracy point. We have numerically confirmed this prediction for $N=4$ particles. In particular we have checked that for $\nu>1 / 2$ (that is for $N=4$ and $\left.N_{\Phi}<8\right)$, no states with zero interaction energy solve the problem at finite spin-orbit coupling strength $q$.

\subsection{At the degeneracy point and slightly above: $3 \leq q^{2} / B \lesssim 3.2$}

Slightly above the degeneracy point (see Fig. 1), when most particles populate the $n=1$ level, the projection of the Laughlin state according to Eq. (19) represents reasonably well the true ground state. As seen in Fig. 2 (a), also this projection yields a gapped state. As in the standard Laughlin state, the ground state is unique (apart from the center-of-mass degeneracy), and characterized by a Haldane momentum $\boldsymbol{K}=\mathbf{0}$. This gives another hint for the Laughlin-like behavior of the system.

The projected Laughlin states, however, fail to describe the system precisely at the degeneracy point. Here, the gap vanishes and a quasi-degenerate manifold of several

states at different $\boldsymbol{K}$ describes the ground state. Apart from the fact that $\boldsymbol{K}=\mathbf{0}$ does not belong to this manifold, the lowest state at this pseudomomentum has no overlap with the projected Laughlin states. The failure of the Laughlin projections at the degeneracy point can be traced back to the fact that the projected Laughlin states are restricted to a single Landau level. We expect that at the degeneracy point, the particles have to be mapped partly according to Eq. (18), and partly according to Eq. (19), but we have no unique prescription for this. At the degeneracy point, our numerical results are very sensitive to particle number with respect to the number and Haldane momenta of the states within the ground state manifold.

\subsection{Clearly above the degeneracy point: $3.2 \lesssim q^{2} / B \lesssim 4$}

The regime where the $n=1$ Laughlin state projection describes well the system turns out to be restricted to a small parameter range above the degeneracy point. When further increasing the spin-orbit coupling strength, the overlap between true ground state and projected Laughlin state rapidly decreases and finally vanishes. For our choice of interaction of Fig. 2 (c), $\mathrm{SU}(2)$ symmetric with $g=0.2$, it is zero already 


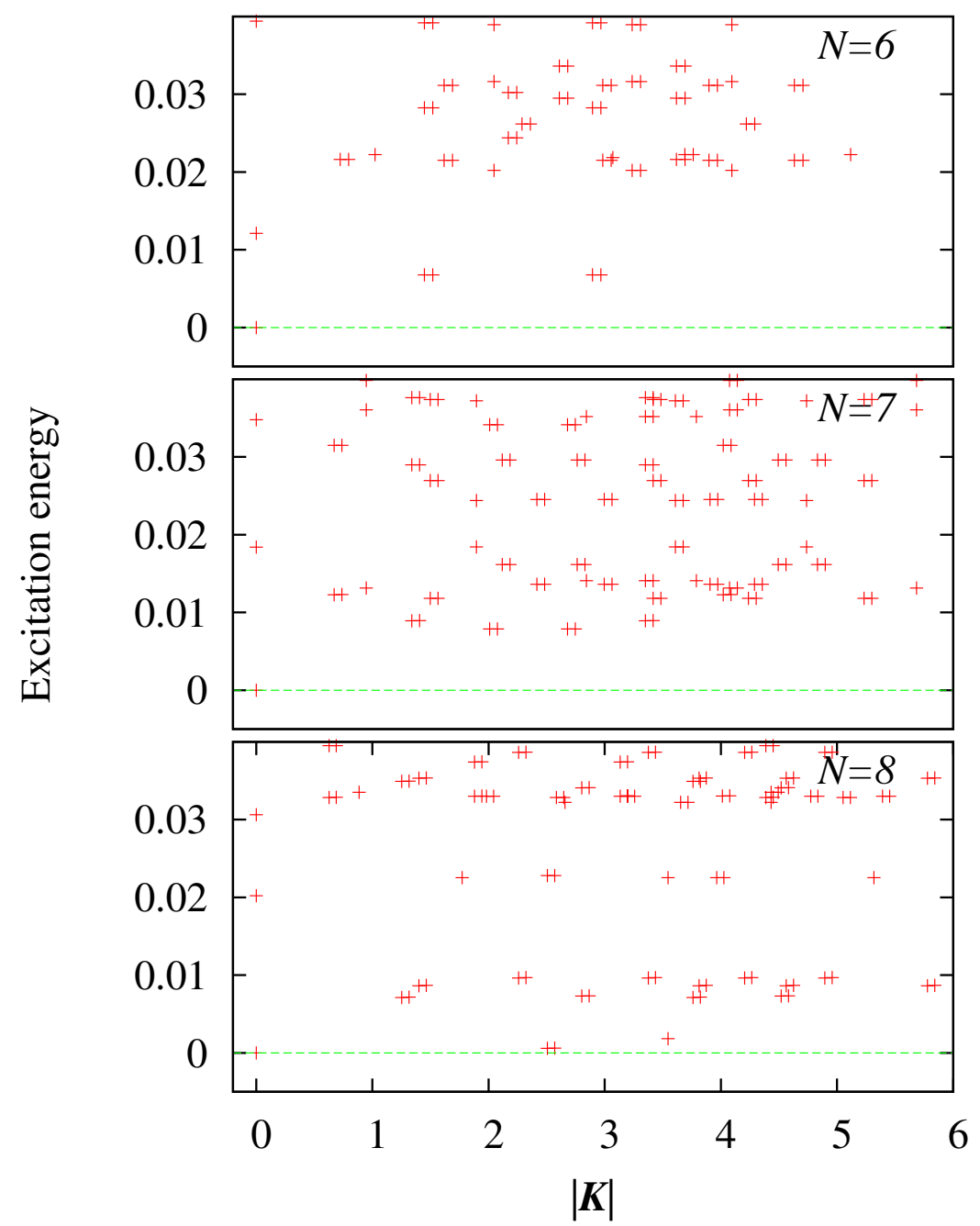

Figure 3. Excitation energies of low-lying states at $q^{2} / B=3.6$ for $\mathrm{SU}(2)$-symmetric interactions $(g=0.2)$. Energies are in units of $g B$, and $|\boldsymbol{K}|$ is in units of $2 \pi \hbar / a$.

for $q^{2} / B=3.2$. Here, the system is still far away from the next degeneracy point at $q^{2} / B=5$, thus the two lowest Landau levels are still given by $n=1$ and $n=0$. Taking into account these two levels, we have performed the exact diagonalization for up to $N=6$ particles. Since in that region, roughly located around $3.2 \lesssim q^{2} / B \lesssim 4$, a significant gap separates the $n=1$ Landau level from the $n=0$ level, we also have performed exact diagonalization studies neglecting the $n=0$ level, which indeed has yielded the same results. With this reduction of the Hilbert space, we have reached system sizes of up to $N=8$ particles.

As before we have considered different interaction parameters. Now the choice with $g_{\uparrow \uparrow}=g_{\downarrow \downarrow}=0$ and $g_{\uparrow \downarrow}>0$ does not lead to a ground state of zero interaction energy in the region above the degeneracy point, since such a state becomes unfavorable with respect to its single-particle energy. The $\mathrm{SU}(2)$-symmetric choice of interactions and the 
configuration with $g_{\uparrow \uparrow}=g_{\downarrow \downarrow}>0$ and $g_{\uparrow \downarrow}=0$ yield qualitative differences in the spectral structure only for $N \leq 5$, which are absent in larger systems. We thus restrict ourselves to presenting the data obtained for the $\mathrm{SU}(2)$-symmetric case.

For all particle numbers, a state at $\boldsymbol{K}=\mathbf{0}$ is the ground state of the system, but this state is not well described by the projected Laughlin state. Neither is it protected by a significant gap: Depending on the particle number, states at different $\boldsymbol{K}$ are energetically very close to the ground state, see Fig. 3. The upper spin component of this state is found to be well described by the ground state of a one-component system which is assumed to live in the first excited Landau level, see Fig. 2 (c). This overlap further increases: For $N=8$ it reaches the value 0.96, if we renormalize the upper spin component to one. Another state which has a sizable overlap with the true ground state is the ground state of a one-component system in the second excited Landau level. Comparing this state with the re-normalized lower spin component of the true ground state, the overlap is 0.38 for $N=6$ and decreases to 0.30 for $N=8$.

The relevance of these states is not very surprising, given the fact that the spin-orbit coupled level $|n=1, j\rangle$ is constructed from the first and second excited Landau level of the spinless problem. But it is noteworthy that the projection of the Laughlin state into these levels has basically no overlap with the respective ground states. Studying the behavior of particles in higher Landau levels is particularly relevant with respect to fermionic systems. In the field of strongly correlated electrons, very much attention has been paid to the Hall plateau at $\nu=5 / 2$. For a spin-polarized system, this represents the situation of a half-filled first excited Landau level, which might be described by a Moore-Read-like wave function [3] and thus support non-Abelian excitations. With bosonic systems, it is not possible to study higher Landau levels by filling the lower levels. However, as our results clearly prove, the spin-orbit coupled system above the degeneracy point behaves with very high fidelity like a one-component system in the first excited Landau level. It thus becomes possible to explore this regime also within bosonic systems.

From the results shown in Fig. 3, it is unclear whether the system develops a gapped phase in the thermodynamic limit, as the tendency observed for sizes up to $N=7$ may suggest. At $N=8$, however, a quasi-degeneracy of the $\boldsymbol{K}=\mathbf{0}$ state with several states at distinct Haldane momenta, indicates that a phase with a broken translational symmetry is established, like crystalline phases or bubble phases [56, 57, 58]. Such phases have been discussed in the context of fermions as possible candidates for substituting the Laughlin state in higher Landau levels.

\subsection{Laughlin states for $\nu<1 / 2$}

The Laughlin state defined in Eq. (25) is characterized by an integer parameter $Q$, which turns out to be the inverse of the filling factor. So far we have discussed only the most prominent case of $Q=2$, but any even value of $Q$ yields a bosonic wave function of zero interaction energy. As has been pointed out in Refs. [46, 47, for $Q>2$ this wave 
function remains a zero interaction energy state when mapped from the lowest Landau level of the system without spin-orbit coupling into the Landau level structure of the spin-orbit coupled system according to Eq. (18). The resulting wave function describes a system with all atoms exclusively in the $|n=0\rangle$ level. It thus represents the ground state for spin-orbit coupling strengths $q^{2} \leq 3 B$ at filling factor $\nu=1 / Q$.

We have explicitly checked for $Q=4$ (and $N=3,4$ ) that such a zero interaction energy state exists for $q^{2} \leq 3 B$. Also, no zero interaction energy states are found for $q^{2} \leq 3 B$ at filling factors larger than $\nu=1 / 4$. In agreement with its Laughlin-like character, it is located at Haldane momentum $\boldsymbol{K}=(0,0)$, and it is the unique groundstate for $q^{2}<3 B$. Precisely at the degeneracy point, $q^{2}=3 B$, additional states with zero interaction energy occur within a ground state manifold which then contains 7 (4) states for $N=4(N=3)$ at $\boldsymbol{K}=(0,0)$, and additional states at different pseudomomenta.

The origin of one of the additional ground states at the degeneracy point can be traced back to the $Q=4$ Laughlin state. Therefore one has to note that every pair of coordinates appears with a $Q$ th order zero of the form $z_{i}-z_{j}$ in the wave function of Eq. (25). Applying the mapping of Eq. (18) to a state contains operations where the original wave function is multiplied with the complex conjugate variable $z_{i}^{*}$, and where the derivatives $\partial_{z_{i}}$ are taken. Only the latter operation may affect the property of being a zero interaction energy state. But since in every term $\left(z_{i}-z_{j}\right)^{Q}$ at most the second derivative $\partial_{z_{i}} \partial_{z_{j}}$ is taken, the mapping of Eq. (18) applied to the wave function of Eq. (25) yields a wave function where every pair of particles still has a zero of at least $(Q-2)$ th order. For $Q=4$, this still allows to raise the Landau level index of one particle from $n=0$ to $n=1$, yielding a partially raised $Q=4$ Laughlin state.

Indeed we find for $q^{2}<3 B$ exactly one state with the total energy $E=$ $(N-1) E_{0}^{-}+E_{1}^{-}$. This state contains one particle in the $n=1$ level, while the others are in $n=0$. It is thus seen to be a state of zero interaction energy. As $E_{1}^{-}>E_{0}^{-}$below the degeneracy point, there it appears as an excited state in the spectrum of the system. The symmetry of this state is characterized by $\boldsymbol{K}=(0,0)$. By lowering the quantum number of the particle in $n=1$ to $n=0$, we have been able to explicitly check that this state is the partially raised Laughlin state.

The other zero interaction energy states occuring at the degeneracy point do not exist in the spectrum for $q^{2}<3 B$. They are not polarized with respect to the Landau level quantum number, that is, not all contributing Fock states contain the same number of particles in each Landau level. Only at the degeneracy point such levels can be combined arbitrarily. This property allows for a larger number of ground states at the degeneracy point.

For larger spin-orbit coupling strengths, $q^{2}>3 B$, the $Q=4$ Laughlin state and its partially raised counterpart remain zero interaction energy eigenstates of the system. However, their single-particle energy now is higher than the lowest possible one given by $N E_{1}^{-}$. This will quickly give rise to a ground state with higher occupation of the $n=1$ level. This state has finite interaction energy, as predicted by Ref. [47]. The precise localization of the transition into the new ground state depends on the interaction 
strength. For the $\mathrm{SU}(2)$ symmetric choice with $g=1$, we get a ground state with finite interaction energy already for $q^{2}=3.03 \sqrt{B}$.

A state of zero interaction energy which is fully polarized in the $n=1$ level is a Laughlin state with $Q=6$ projected into $n=1$. At such a low filling, $\nu=1 / 6$, our numerics is restricted to $N=3$ particles. Of course, a lot of states with zero interaction energy exist at this filling, but only one has the property of being completely within the $n=1$ level. Such state is not found at higher filling, that is for $N=3$ and $N<N_{\Phi}=18$. Obviously, this state is the true ground state between the first and the second degeneracy point. If we consider, instead of the $\mathrm{SU}(2)$ symmetric choice, an interaction only between different pseudospins, that is $g_{\uparrow \uparrow}=g_{\downarrow \downarrow}=0$ and $g_{\uparrow \downarrow}>0$, zero interaction energy states within the $n=1$ level have been predicted for fillings up to $\nu=1 / 4$ [47]. We have checked this prediction for $N=4$. Dor spin-orbit coupling strengths $q$ which are sufficiently far above the degeneracy point, the unique ground state, with Haldane symmetry $\boldsymbol{K}=(0,0)$, is given by this fully polarized state of zero interaction energy.

\section{Incompressible phases at the first degeneracy point}

We have seen in the previous section that above and below the degeneracy point the system behaves as an incompressible Laughlin liquid at $\nu=1 / Q$ with $Q$ even. As we have shown in most detail for $\nu=1 / 2$, the incompressibility is lost at the degeneracy point, where the number of states in the lowest Landau level is doubled, giving rise to a degeneracy also on the many-body level. In this section we investigate the system at this degeneracy point, and look for filling factors which might support gapped phases. All results presented below have been obtained for an $\mathrm{SU}(2)$ symmetric choice of interactions.

\section{1. $\nu=2$}

A clearly gapped phase shows up at filling $\nu=2$, where a unique ground state at $\boldsymbol{K}=\mathbf{0}$ is found for $N=8$ and $N=10$. This fact makes this phase distinct from the ReadRezayi states and the NASS states which are both possible candidates due to the filling factor. Apart from the large gap, the robustness of the spectrum against deformations of the torus ratio, shown in Fig. 4, signals the incompressibility of this phase.

\section{2. $\nu=3 / 2$}

As has been pointed out in several works [46, 49, 50], off the degeneracy points incompressible phases are supported not only at $\nu=1 / 2$, but also at other fillings corresponding to the Read-Rezayi series [48]. At the Read-Rezayi filling, $\nu=3 / 2$ for $N=6$, a ground-state at $\boldsymbol{K}=\mathbf{0}$ is separated by a sizable gap of about twice the typical energy difference from a second state at $\boldsymbol{K}=\mathbf{0}$. Since a two-fold degenerate ground state at $\boldsymbol{K}=\mathbf{0}$ characterizes the $\nu=3 / 2$ Read-Rezayi state on the torus, one might 


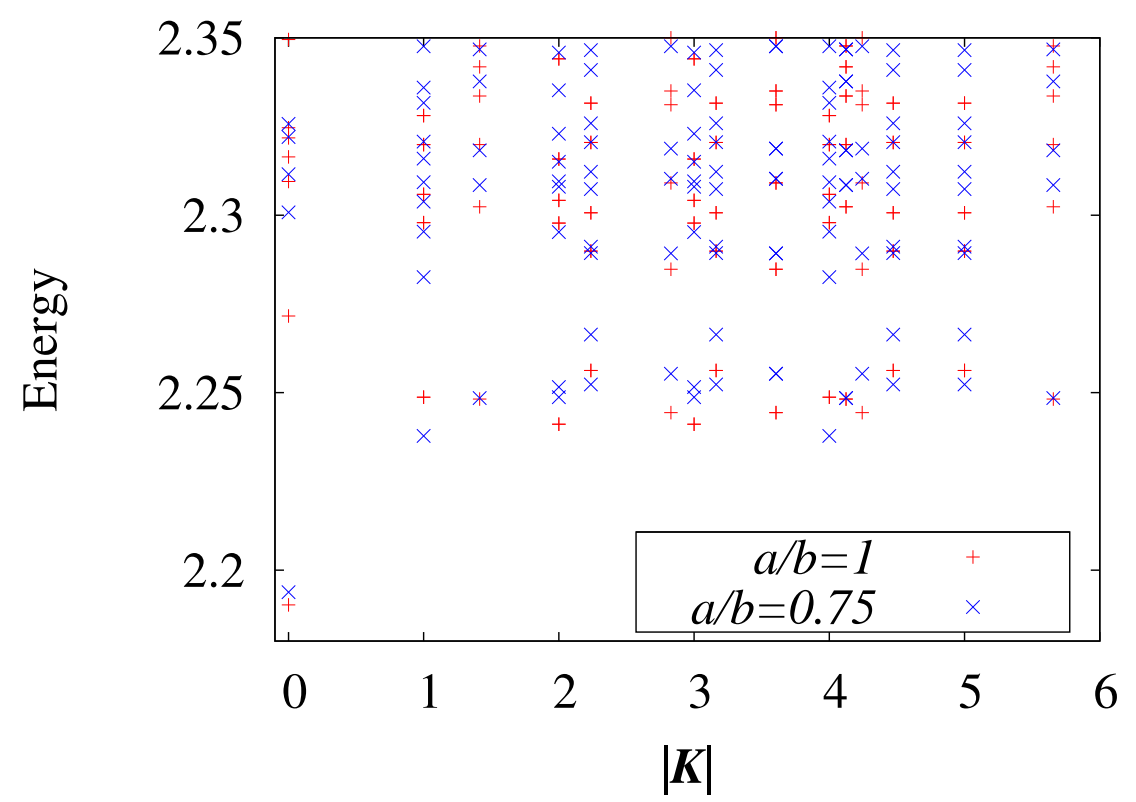

Figure 4. Spectrum at $\nu=2$ for $\mathrm{SU}(2)$ symmetric interactions. Axis ratios 1 and 0.75 are plotted. Note that the degeneracy between $90^{\circ}$ rotations in $\boldsymbol{K}$-space is lifted for ratios different from 1 , and therefore, more states seem to be present at ratio 0.75 . Energies in units of $g B$, and $|\boldsymbol{K}|$ in units of $2 \pi \hbar / a$.

expect that at this filling a Read-Rezayi phase exists on the degeneracy point. We have thus calculated the ground-states of a spin-polarized system with four-body contact interaction. Projecting the two zero-energy states into the Landau level structure of the spin-orbit coupled system, we obtain an overlap of 0.70 with the real ground state. This number is close to the total weight of the fully polarized contribution to this state with all atoms in the $n=0$ level, being 0.72 . For the second state, an overlap of 0.32 is found. Increasing the particle number to $N=9$, we still find overlaps of 0.39 and 0.42 , but the spectral structure is not robust. While one state at $\boldsymbol{K}=\mathbf{0}$ remains the ground state, the second state at this pseudomomentum lies above other states in the spectrum.

\section{3. $\nu=1$}

In spin-polarized Bose systems, the famous Moore-Read state is supported at filling factor $\nu=1$. Also for spin-orbit coupled systems, this state plays a role: Below the degeneracy point $\left(q^{2}<3 B\right)$, the three lowest eigenstates are found at $\boldsymbol{K}=\left(N_{\Phi} / 2,0\right)$, $\left(0, N_{\Phi} / 2\right)$, and $\left(N_{\Phi} / 2, N_{\Phi} / 2\right)$. The phase can thus be identified with the Moore-Read phase [49]. However, this spectral structure disappears at the degeneracy point. Here we find ground states at different pseudomomenta, which even depend on the size of system: $(3,3),(3,5),(5,3),(5,5),(4,0)$, and $(0,4)$ for $N=5$, and $(3,0),(0,3),(2,2)$, $(2,4),(4,2)$, and $(4,4)$ for $N=6$. For $N=7$, states at $(2,0),(5,0),(0,2)$, and $(0,5)$ provide the ground state manifold. For $N=8$, we have $(3,3),(3,5),(5,3),(5,5),(4,0)$, 

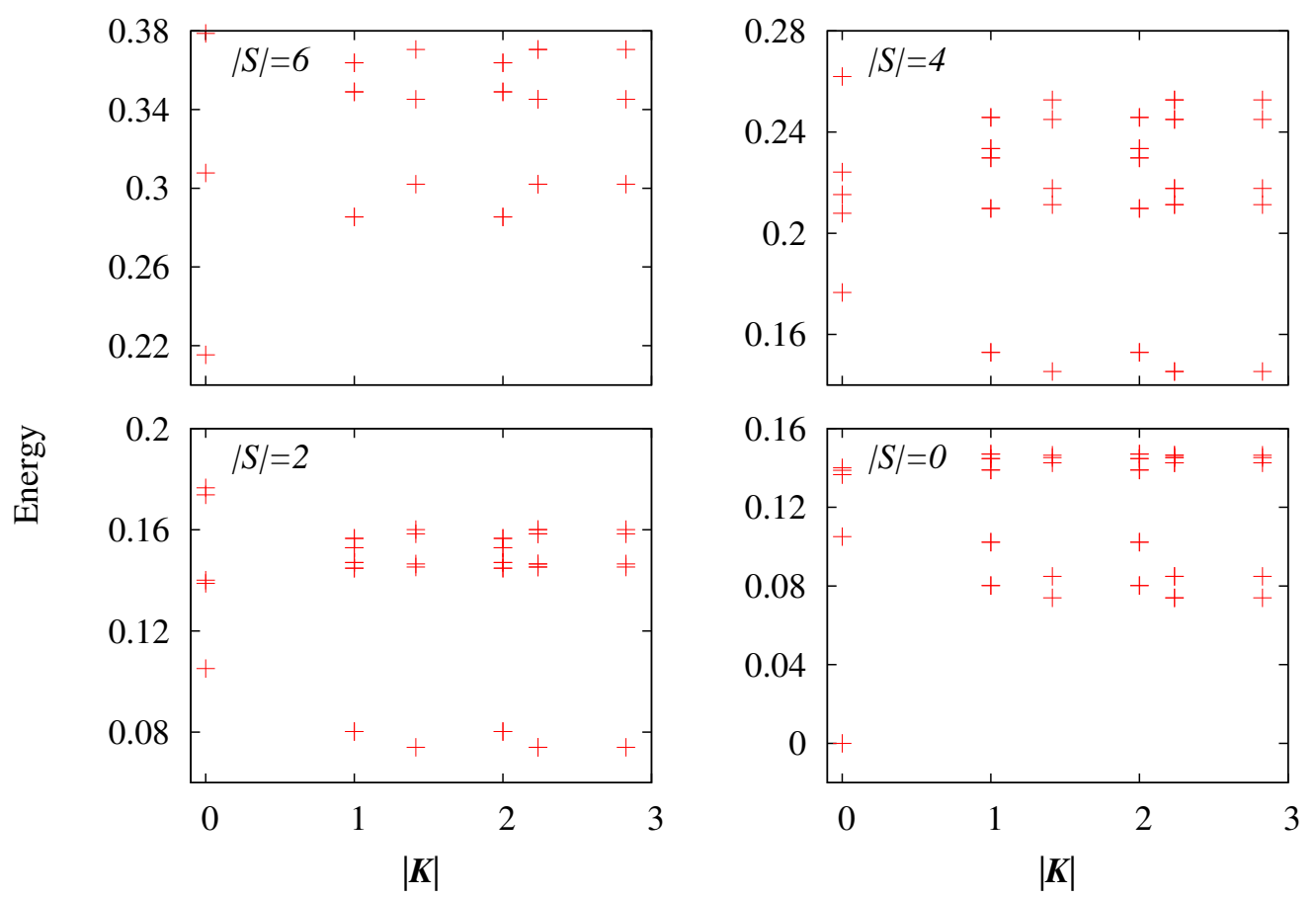

Figure 5. Spectra of a two-component Bose gas without spin-orbit coupling $(q=0)$ at filling factor $\nu=2 / 3$ for different spin polarizations $S . N=6$.

and $(0,4)$. Since the gap between these states, compared to other energy differences in the spectrum, is not extraordinarily big, the system is expected to be compressible.

\subsection{NASS series}

The NASS filling factors, $\nu=2 / 3$ and $\nu=4 / 3$, have been recently shown to support incompressible phases in the system without spin-orbit coupling [42, 43]. It is instructive to recall the results in that limit, $q=0$, for our subsequent discussion at the first degenerate point. In Fig. 5 we depict the spectrum of a system of six atoms interacting through a two-body contact interaction at $q=0$ at filling $\nu=2 / 3$. While for $|S|=6$ and $|S|=0$ a state with $\boldsymbol{K}=\mathbf{0}$ is the unique ground state, a (quasi)degenerate manifold of states with $\boldsymbol{K} \neq \mathbf{0}$ provides the ground state for spin polarizations $|S|=2$ and $|S|=4$. Interestingly, the energy of low-lying excitations at $|S|$ precisely agree with the ground state energy at $\left|S^{\prime}\right|=|S|+2$.

Similarly, for the $\nu=4 / 3$ filling, we have compared the spectrum obtained for a two-body contact interaction with the one obtained with only three-body contact interactions. Both spectra are depicted in Fig. 6. The experimentally feasible case of two-body contact interactions shares most of the spectral properties found in the threebody contact case in all polarizations. In particular, it is especially telling to note that 

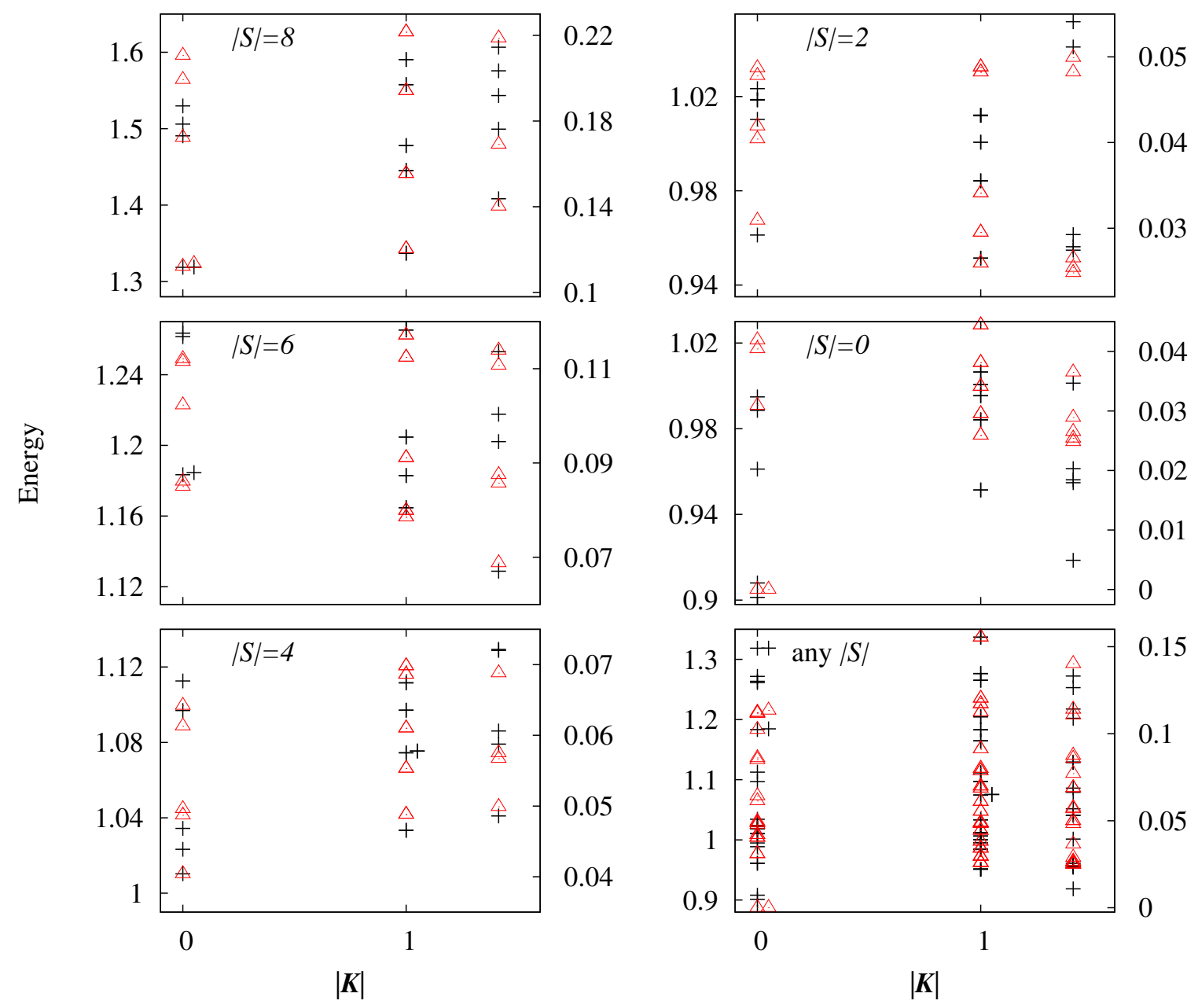

Figure 6. Spectra of a two-component Bose gas without spin-orbit coupling $(q=0)$ at filling factor $\nu=4 / 3$ for different spin polarizations $S$. The results for a two-body contact interaction are given by the black crosses which correspond to the energy scale on the left. The red triangles with the energy scale on the right show the results for a system interacting via a three-body contact potential.

the degeneracies of the lowest states for each polarization are shared in both calculations, a signal of the topological equivalence between both systems [4]. For $S=0$, the gapped ground state of the three-body contact interaction defines a NASS phase.

Now, we turn back to the spin-orbit coupled system. Here we do find a clear gap in the spectrum at $\nu=2 / 3$ with $N=6$, and a small gap at $\nu=4 / 3$ with $N=8$, see Fig. 7 ,

For the $\nu=2 / 3$ filling, the nature of the ground state of the system can be related to the 221-Halperin state, Eq. (44). We have projected the 221-Halperin state, onto the Landau level structure of the spin-orbit coupled system according to Eq. (18), and computed the overlap of this state with the $\nu=2 / 3$ ground state. We find the value of 0.18. It should be noted, however, that interactions now can induce "spin" flips within 

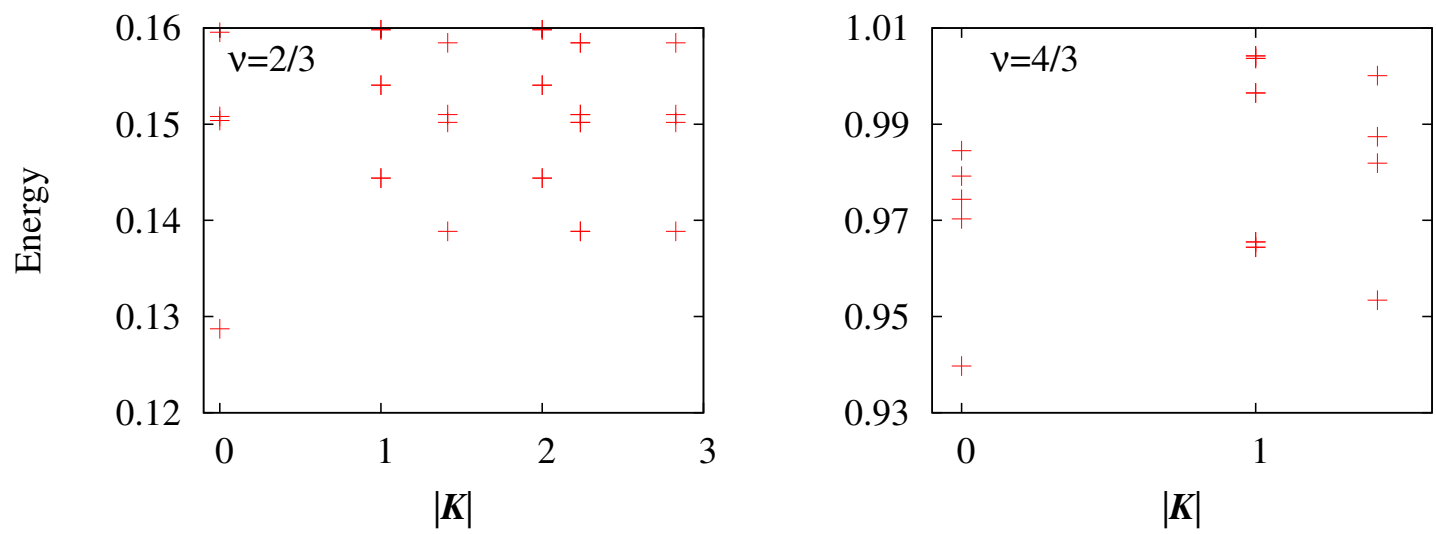

Figure 7. Spectrum at the degeneracy point $q^{2} / B=3$ for filling factor $\nu=2 / 3$ with $N=6$ (left) and for $\nu=4 / 3$ with $N=8$ (right). We have chosen $\mathrm{SU}(2)$ symmetric interactions. Energies in units of $g B$, and $|\boldsymbol{K}|$ in units of $2 \pi \hbar / a$.

the two-fold degenerate manifold. Therefore the analog of spin polarization, namely population imbalance $p \equiv N_{0}-N_{1}$, is not conserved. Here, $N_{1}\left(N_{0}\right)$ define the number of particles in the $n=1(n=0)$ Landau level. We therefore write the true ground state $|\Psi\rangle$ as

$$
|\Psi\rangle=\sum_{i=-N}^{N} w_{i}|p=i\rangle_{\Psi},
$$

with $|p=i\rangle_{\Psi}$ the contributions with well-defined population imbalance. With a proper choice for the phase of each $|p=i\rangle_{\Psi}$, we can make $w_{i}$ a real number, measuring the weight of each polarization $p$. Mapping the Halperin state with spin polarization $S=0$ to the spin-orbit coupled Landau levels according to Eq. (18) yields a state with $p=0$. The overlap of this state with $|\Psi\rangle$ thus is bounded by the corresponding weight, $w_{0}=0.26$. Contrarily, the overlap of the projected Halperin state with $|p=0\rangle_{\Psi}$ takes a significantly increased value, $0.18 / 0.26 \approx 0.7$.

Interestingly, the most important contributions to $|\Psi\rangle$ are $|p=6\rangle_{\Psi}$ with a weight $w_{6}=0.64,|p=4\rangle_{\Psi}$ with a weight $w_{4}=0.57$, and $|p=2\rangle_{\Psi}$ with a weight $w_{2}=0.42$. The contributions with $p<0$ are rarely populated with weights $w_{-2}=0.1, w_{-4}=0.07$, and $w_{-6}=0.01$. This shows that population of $n=0$ is still favored, despite the degeneracy with $n=1$ on the single-particle level. It reflects a fact which we have already encountered before when analyzing the system at $\nu=1 / 2$ : Contact interactions favor the Landau level with lower $n$.

As mentioned above, with good overlap we can interpret the $p=0$ contribution as a projected Halperin state, that is, as the ground state of a two-component system without spin-orbit coupling $(q=0)$ in a two-body contact potential with spin polarization $S=0$ and filling factor $\nu=2 / 3$. Diagonalizing such a system for different $S$, and projecting the lowest state with $\boldsymbol{K}=\mathbf{0}$ according to Eq. (18), we are able to relate all the states $|p=i\rangle_{\Psi}$ in the decomposition of Eq. (26) to ground states of the two-body contact potential for a fixed spin polarization $S$ and Haldane momentum $\boldsymbol{K}$. Explicitly, we find 
an overlap of 0.94 between the projected ground state at $S=6$ and $|p=6\rangle_{\Psi}$, an overlap of 0.84 for $S=4$, and an overlap of 0.83 for $S=2$. Modeling a state with the projected ground states for all spin polarizations $-6 \leq S \leq 6$, we are able to reproduce the true ground state $|\Psi\rangle$ with a fidelity of 0.82 .

For $\nu=4 / 3$ with $N=8$, the overlap with the projected NASS state is only 0.03 . Nevertheless, the true ground state can be reproduced from the lowest $\boldsymbol{K}=\mathbf{0}$ states of a three-body contact interaction, if we take into account different spin polarizations $S$ and project them according to Eq. (18). With this we are able to model the true ground state of the spin-orbit coupled system with a total fidelity of 0.52 .

\subsection{Low filling}

Finally we note that an incompressible phase can be expected at a critical $\nu_{0}$, defined as the largest filling supporting states with zero interaction energy. For any $\nu<\nu_{0}$, the interaction energy should remain zero, while for $\nu>\nu_{0}$ it is by definition larger than zero. Thus, at $\nu_{0}$ a kink in the energy as a function of $\nu$ can be expected, resulting in an incompressibility of the system. As Fig. 2 (b) shows, the spin-orbit coupled system with two-body contact interaction has a finite interaction energy at filling $\nu=1 / 2$, in contrast to the system with only an Abelian gauge field. This is a consequence of the fact that, at this filling, there are no zero-energy states of two-body contact interaction within the first excited or higher Landau levels. We thus should focus on smaller filling factors.

By analytical arguments, a zero interaction energy state of the form of a (464) Halperin state has been predicted in Ref. [47]. With half of the particles in the $n=0$ level, and half of the particles in the $n=1$ level, this state has been constructed as an unpolarized state with $p=0$. However, such a state is ill-defined on a torus: The filling factor of the $n=0$ level is given as $\nu_{n=0}=1 /(4+4)=1 / 8$, while for $n=1$ it reads $\nu_{n=1}=1 /(4+6)=1 / 10$. Thus, the state cannot have $p=0$, if both levels are assumed to fill the same area.

However, for arbitrary $p$, we are able to find states of zero interaction energy at even larger filling factors. As already mentioned in Sec. 5.4, also $\nu=1 / 4$ supports states with zero interaction energy. Apart from the Laughlin state $(p=N)$ and a Laughlin state with one particle shifted into the first excited Landau level $(p=N-2)$, which are present in the spectrum of the system at any $q$, additional zero-interaction appear at the degeneracy point. For $N=4(N=3)$, there are 5 (2) additional zero interaction energy states with $K=(0,0)$. Further states with zero interaction energy appear at finite pseudomomenta. For $N=3$, there are two such states for each pseudomomenta, while for $N=4$ this number varies between 4 and 5 . These zero interaction energy states do not exist for $q^{2}<3 B$.

The huge degeneracy of zero interaction energy eigenstates at $\nu=1 / 4$ suggests that this is not yet the critical filling $\nu_{0}$ at the degeneracy point. In fact, we find a unique zero interaction energy state at $\boldsymbol{K}=(0,0)$ for $\nu=2 / 7$ and $N=4$. This state 
has no well defined polarization $p$, but the large weight $w_{4}=0.9298$ indicates that the atoms are mostly in the lowest Landau level, $n=0$. However, this makes the state to be an eigenstate only at the degeneracy point, leaving the Laughlin filling $\nu=1 / 4$ to be the critical filling off the degeneracy point. We do not find states with zero interaction energy are for $\nu=1 / 3$ (for $N=4$ and $N=5$ ) or $\nu=4 / 13$ (for $N=4$ ). Here, we therefore expect $\nu=2 / 7$ to be the critical filling of the system.

This would shift the incompressible phase from $\nu=1 / 4$ for $q^{2}<3 B$ to $\nu=2 / 7$ for $q^{2}=3 B$. However, we should also note that the excitation gap above the ground state at $\nu=2 / 7$ state takes the tiny value of $2 \cdot 10^{-4} \mathrm{gB}$. The energy as a function of $\nu$ is a concave function, and in the thermodynamic limit it might happen that $\frac{\mathrm{d} E}{\mathrm{~d} \nu} \rightarrow 0$, when $\nu \searrow \nu_{0}$. This would render a compressible phase even at $\nu_{0}$. We therefore conclude that, at the degeneracy point, robust incompressible phases are rather located in the denser regime. This should facilitate the experimental observation.

\section{Summary and conclusions}

We have performed a numerical study of the ground and excited states of an ultracold pseudospin $1 / 2$ bosonic cloud subjected to an artificial magnetic field. The setup considered, first proposed in Ref. [46], includes a spin-orbit coupling, which is mathematically equivalent to a non-Abelian SU(2) gauge potential. The single particle spectrum has a number of degenerate points which have received a special attention, as two different Landau levels simultaneously provide the low-energy manifold of singleparticle states. This fact makes them particularly appealing for exploring interesting new phases.

We have concentrated on the parameter region around the first degenerate point $q^{2} / B=3$. First, we have analyzed $\nu=1 / 2$ and discussed the different Laughlin-like states which have a large overlap with the ground state of the system. The exact ground state of the system at this filling is described either by the usual Laughlin wave function (mapped into our Landau level structure), if we are below the degenerate point, or by the corresponding ground state of contact interactions in the first excited Landau level, for values of $q$ above the degenerate point. This allows to explore higher Landau levels even with bosons. Slightly above the degenerate point there is a sizable overlap between the ground state and a Laughlin state projected into the first excited Landau level. At filling $\nu=1 / 4$, we find two Laughlin-like states with zero interaction energy even in the spin-orbit coupled system. One of them is the ground state for $q^{2} / B<3$. Additional states of zero interaction energy are found at the degeneracy point for $\nu=1 / 4$, and it turns out that the critical filling is shifted to $\nu=2 / 7$. Above the degeneracy point, ground states with zero interaction energy are found only for $\nu=1 / 6$. An exception are configurations without intraspecies interactions.

Secondly, we have studied in detail the phases appearing at the first degenerate point for the most interesting filling factors. Notably, we find an unexpected gapped phase at $\boldsymbol{K}=\mathbf{0}$ at a fairly dense filling, $\nu=2$. This phase is gapped for $N=8$ and 
$N=10$ and is robust for changes in the aspect ratio of the torus, as expected for an incompressible phase. Motivated by the $\nu=1 / 2$ results, we looked for incompressible phases at other Read-Rezayi fillings, $\nu=1$ and $\nu=3 / 2$. For $\nu=1$ we find no indication of a gap in the spectrum of the system. For the $\nu=3 / 2$ we find a sizable overlap between the exact ground state of the system and the ground state obtained with four-body contact interactions mapped into our Landau level structure. The spectral structure however is not robust as we increase the particle number, finding that the two-fold degeneracy of the $\boldsymbol{K}=\mathbf{0}$ ground state is lifted when we go from six to nine particles.

Thirdly, we have analyzed the gapped phases occuring at the NASS filling factors $\nu=2 / 3$ and $\nu=4 / 3$. Decomposing the ground states into the contributions with a fixed population imbalance between the two Landau levels, we are able to relate these states to the ground states of a two-body contact potential for $\nu=2 / 3$, or of a three-body contact potential for $\nu=4 / 3$, obtained for fixed spin polarizations in a system without spin-orbit coupling.

As a conclusion, we note that the properties of the spin-orbit coupled system in the vicinity of the degeneracy point make the considered setup very appealing from the experimental point of view: Upon tuning the spin-orbit coupling strength, transitions between different topological phases can be realized. It also becomes possible to explore the behavior of bosons in higher Landau levels. For the relatively dense system at filling $\nu=2$, a robust gapped phase occurs which is neither described by the NASS nor the Read-Rezayi series.

\section{Acknowledgments}

The authors thank Nuria Barberán for interesting discussions on the "fate of the Laughlin" state, Sec. 5. This work has been supported by EU (NAMEQUAM, AQUTE), ERC (QUAGATUA), Spanish MINCIN (FIS2008-00784 TOQATA), Alexander von Humboldt Stiftung, and AAII-Hubbard. B. J.-D. is supported by the Ramón y Cajal program. M. L. acknowledges support from the Joachim Herz Foundation and Hamburg University.

\section{Appendix A. Interaction matrix elements}

The interaction matrix elements $V_{\{n, j\}}$, with $\{n, j\}$ denoting the set of quantum numbers $n_{1}, \ldots, n_{4}$ and $j_{1}, \ldots, j_{4}$, are given by

$$
V_{\{n, j\}}=\left\langle n_{1}, j_{1}\left|\left\langle n_{2}, j_{2}|V| n_{3}, j_{3}\right\rangle\right| n_{4}, j_{3}\right\rangle,
$$

with $V$ given in Eq. (3i). This yields:

$$
\begin{aligned}
V_{\{n, j\}} & =\frac{1}{2}\left(g_{\uparrow \uparrow} \alpha_{n_{1}}^{-} \alpha_{n_{2}}^{-} \alpha_{n_{3}}^{-} \alpha_{n_{4}}^{-} \mathcal{I}_{n_{1}, n_{2}, n_{3}, n_{4}}^{j_{1}, j_{2}, j_{3}, j_{4}}\right. \\
& +g_{\downarrow \downarrow} \beta_{n_{1}}^{-} \beta_{n_{2}}^{-} \beta_{n_{3}}^{-} \beta_{n_{4}}^{-} \mathcal{I}_{n_{1}+1, n_{2}+1, n_{3}+1, n_{4}+1}^{j_{1}, j_{2}, j_{3}, j_{4}}
\end{aligned}
$$


FQH states of a Bose gas with spin-orbit coupling

$$
\begin{aligned}
& +g_{\uparrow \downarrow} \alpha_{n_{1}}^{-} \beta_{n_{2}}^{-} \alpha_{n_{3}}^{-} \beta_{n_{4}}^{-} \mathcal{I}_{n_{1}, n_{2}+1, n_{3}, n_{4}+1}^{j_{1}, j_{2}, j_{3}, j_{4}} \\
& \left.+g_{\downarrow \uparrow} \beta_{n_{1}}^{-} \alpha_{n_{2}}^{-} \beta_{n_{3}}^{-} \alpha_{n_{4}}^{-} \mathcal{I}_{n_{1}+1, n_{2}, n_{3}+1, n_{4}}^{j_{1}, j_{2}, j_{3}, j_{4}}\right) \text {, }
\end{aligned}
$$

with

$$
\begin{aligned}
\mathcal{I}_{\{n\}}^{\{j\}} & =\int_{0}^{a} \int_{0}^{b} \mathrm{~d}^{2} \boldsymbol{r}_{1} \mathrm{~d}^{2} \boldsymbol{r}_{2} \delta\left(\boldsymbol{r}_{1}-\boldsymbol{r}_{2}\right) \Psi_{n_{1} j_{1}}^{*}\left(\boldsymbol{r}_{1}\right) \Psi_{n_{2} j_{2}}^{*}\left(\boldsymbol{r}_{2}\right) \\
& \times \Psi_{n_{3} j_{3}}\left(\boldsymbol{r}_{1}\right) \Psi_{n_{4} j_{4}}\left(\boldsymbol{r}_{2}\right) .
\end{aligned}
$$

To evaluate this integral, we write the elliptic $\theta$ functions in $\Psi_{n j}$ as infinite sums, see also [55, 59]. We get

$$
\begin{aligned}
\mathcal{I}_{\{n\}}^{\{j\}} & =\frac{1}{2 \pi N_{\Phi}} \sum_{\mu=-\infty}^{\infty} \sum_{\nu=-\infty}^{\infty} \delta_{j_{1}+j_{2}, j_{3}+j_{4}}^{\prime} \delta_{j_{1}-j_{4}, t}^{\prime} \\
& \times C_{n_{1}, n_{4}}\left(\frac{\tau}{\vartheta} \mu, \tau \vartheta \nu\right) C_{n_{2}, n_{3}}\left(-\frac{\tau}{\vartheta} \mu,-\tau \vartheta \nu\right) \\
& \times \exp \left[-\tau^{2}\left\{\frac{1}{2}\left(\left(\frac{\mu}{\vartheta}\right)^{2}+(\nu \vartheta)^{2}\right)+i \mu\left(j_{1}-j_{3}\right)\right\}\right] .
\end{aligned}
$$

where $\delta^{\prime}$ is a Kronecker delta modulo $N_{\Phi}, \tau=\sqrt{2 \pi / N_{\Phi}}$, and $\vartheta=\sqrt{a / b}$. The coefficients $C_{n_{i}, n_{j}}$ read (with $u, v \in \mathbb{R}$ ):

$$
\begin{aligned}
& C_{1,0}(u, v)=-(u+i v) / \sqrt{2}=-C_{0,1}^{*}(u, v), \\
& C_{1,1}(u, v)=1+C_{1,0}(u, v) C_{0,1}(u, v), \\
& C_{2,0}(u, v)=\sqrt{2}\left[C_{0,1}(u, v)\right]^{2}=-C_{0,2}^{*}(u, v), \\
& C_{1,2}(u, v) \equiv\left(u^{2}+v^{2}-4\right)(u+i v) / 4=-C_{2,1}^{*}(u, v), \\
& C_{2,2}(u, v) \equiv\left(8-8\left(u^{2}+v^{2}\right)+2 u^{2} v^{2}+u^{4}+v^{4}\right) / 8 .
\end{aligned}
$$

The sums in Eq. (A.4) converge quickly, especially within the lowest Landau level. For levels up to $n=2$, taking into account only values with $|\mu| \leq 16$ and $|\nu| \leq 8$ provides very precise values. 
[1] Laughlin R B 1983 Phys. Rev. Lett. 501395

[2] Arovas D, Schrieffer J R and Wilczek F 1984 Phys. Rev. Lett. 53(7) 722-723

[3] Moore G and Read N 1991 Nucl. Phys. B 360362

[4] Wen X G and Niu Q 1990 Phys. Rev. B 41(13) 9377-9396

[5] Cooper N 2008 Adv. Phys. 57539

[6] Fetter A L 2009 Rev. Mod. Phys. 81 647-691

[7] Cooper N R and Wilkin N K 1999 Phys. Rev. B 60(24) R16279-R16282

[8] Wilkin N K and Gunn J M F 2000 Phys. Rev. Lett. 84(1) 6-9

[9] Cooper N R, Wilkin N K and Gunn J M F 2001 Phys. Rev. Lett. 87(12) 120405

[10] Schweikhard V, Coddington I, Engels P, Mogendorff V P and Cornell E A 2004 Phys. Rev. Lett. 92040404

[11] Matthews M R, Anderson B P, Haljan P C, Hall D S, Wieman C E and Cornell E A 1999 Phys. Rev. Lett. 83(13) 2498-2501

[12] Schweikhard V, Coddington I, Engels P, Tung S and Cornell E A 2004 Phys. Rev. Lett. 93(21) 210403

[13] Rosenbusch P, Petrov D S, Sinha S, Chevy F, Bretin V, Castin Y, Shlyapnikov G and Dalibard J 2002 Phys. Rev. Lett. 88(25) 250403

[14] Bretin V, Stock S, Seurin Y and Dalibard J 2004 Phys. Rev. Lett. 92(5) 050403

[15] Dalibard J, Gerbier F, Juzeliūnas G and Öhberg P 2011 Rev. Mod. Phys. 83(4) 1523-1543

[16] Juliá-Díaz B, Dagnino D, Günter K J, Graß T, Barberán N, Lewenstein M and Dalibard J 2011 Phys. Rev. A 84(5) 053605

[17] Juliá-Díaz B, Graß T, Barberán N and Lewenstein M 2012 New J. Phys. 14055003

[18] Lin Y J, Compton R L, Perry A R, Phillips W D, Porto J V and Spielman I B 2009 Phys. Rev. Lett. 102130401

[19] Lin Y J, Compton R L, Jiménez-García K, Porto J V and Spielman I B 2009 Nature 462628

[20] Jiménez-García K, LeBlanc L J, Williams R A, Beeler M C, Perry A R and Spielman I B 2012 Phys. Rev. Lett. 108(22) 225303

[21] Aidelsburger M, Atala M, Nascimbène S, Trotzky S, Chen Y A and Bloch I 2011 Phys. Rev. Lett. $\mathbf{1 0 7}(25) 255301$

[22] Struck J, Ölschläger C, Weinberg M, Hauke P, Simonet J, Eckardt A, Lewenstein M, Sengstock K and Windpassinger P 2012 Phys. Rev. Lett. 108(22) 225304

[23] Bychkov Y A and Rashba E I 1984 J. Phys. C 176039

[24] Juzeliūnas G and Öhberg P 2004 Phys. Rev. Lett. 93033602

[25] Ruseckas J, Juzeliūnas G, Öhberg P and Fleischhauer M 2005 Phys. Rev. Lett. 95010404

[26] Stanescu T D, Anderson B and Galitski V 2008 Phys. Rev. A 78(2) 023616

[27] Lin Y J, Jiménez-García K and Spielman I B 2011 Nature 471 83-86

[28] Williams R A, LeBlanc L J, Jiménez-García K, Beeler M C, Perry A R, Phillips W D and Spielman I B 2012 Science 335 314-317

[29] Sinha S, Nath R and Santos L 2011 Phys. Rev. Lett. 107(27) 270401

[30] Hu H, Ramachandhran B, Pu H and Liu X J 2012 Phys. Rev. Lett. 108(1) 010402

[31] Barnett R, Powell S, Graß T, Lewenstein M and Das Sarma S 2012 Phys. Rev. A 85(2) 023615

[32] Barnett R, Powell S, Graß T, Lewenstein M and Das Sarma S 2012 Phys. Rev. A 85(4) 049905

[33] Ozawa T and Baym G 2012 Phys. Rev. Lett. 109(2) 025301

[34] Ozawa T and Baym G 2012 Phys. Rev. A 85(6) 063623

[35] Li Y, Pitaevskii L P and Stringari S 2012 Phys. Rev. Lett. 108(22) 225301

[36] Osterloh K, Baig M, Santos L, Zoller P and Lewenstein M 2005 Phys. Rev. Lett. 95010403

[37] Hauke P, Tieleman O, Celi A, Ölschläger C, Simonet J, Struck J, Weinberg M, Windpassinger P, Sengstock K, Lewenstein M and Eckardt A 2012 Phys. Rev. Lett. 109(14) 145301

[38] Graß T, Saha K, Sengupta K and Lewenstein M 2011 Phys. Rev. A 84(5) 053632

[39] Cole W S, Zhang S, Paramekanti A and Trivedi N 2012 Phys. Rev. Lett. 109(8) 085302

[40] Radić J, Di Ciolo A, Sun K and Galitski V 2012 Phys. Rev. Lett. 109(8) 085303 
[41] Graß T, Baranov M A and Lewenstein M 2011 Phys. Rev. A 84(4) 043605

[42] Graß T, Juliá-Díaz B, Barberán N and Lewenstein M 2012 Phys. Rev. A 86(2) 021603

[43] Furukawa S and Ueda M 2012 Phys. Rev. A 86(3) 031604

[44] Ardonne E and Schoutens K 1999 Phys. Rev. Lett. 82(25) 5096-5099

[45] Ardonne E, Read N, Rezayi E and Schoutens K 2001 Nucl. Phys. B 607 549-576

[46] Burrello M and Trombettoni A 2010 Phys. Rev. Lett. 105125304

[47] Burrello M and Trombettoni A 2011 Phys. Rev. A 84(4) 043625

[48] Read N and Rezayi E 1999 Phys. Rev. B 59(12) 8084-8092

[49] Palmer R N and Pachos J K 2011 New J. Phys. 13065002

[50] Komineas S and Cooper N R 2012 Phys. Rev. A 85(5) 053623

[51] Zhang Y, Mao L and Zhang C 2012 Phys. Rev. Lett. 108(3) 035302

[52] Halperin B I 1983 Helv. Phys. Acta 5675

[53] Yoshioka D, Halperin B I and Lee P A 1983 Phys. Rev. Lett. 50(16) 1219-1222

[54] Haldane F D M 1985 Phys. Rev. Lett. 55(20) 2095-2098

[55] Chakraborty T and Pietilainen P 1995 The Quantum Hall Effects (Springer)

[56] Fogler M M and Koulakov A A 1997 Phys. Rev. B 55(15) 9326-9329

[57] Haldane F D M, Rezayi E H and Yang K 2000 Phys. Rev. Lett. 85(25) 5396-5399

[58] Grusdt F and Fleischhauer M 2012 arXiv:1207.3716

[59] Výborný K 2005 Spin in fractional quantum Hall systems Ph.D. thesis University of Hamburg 